"This is an Accepted Manuscript of an article published by Macromol. Theory and Simul., mats.201700088, accepted in December 2018; vol 27, no 2, 1700088 (2018)."

\title{
Heuristic Search Strategy for Transforming Microstructural Patterns to Optimal Copolymerization Recipes
}

Yousef Mohammadi ${ }^{1 *}$, Mohammad Reza Saeb², Alexander Penlidis ${ }^{3 *}$

1 Petrochemical Research and Technology Company (NPC-rt), National Petrochemical Company (NPC), P.O. Box 14358-84711, Tehran, Iran

2 Department of Resin and Additives, Institute for Color Science and Technology, P.O. Box 16765-654, Tehran, Iran

${ }^{3}$ Department of Chemical Engineering, Institute for Polymer Research (IPR), University of Waterloo, Waterloo, Ontario N2L 3G1 Canada

To whom correspondence should be addressed:

Dr. Yousef Mohammadi: mohammadi@npc-rt.ir

Prof. Alexander Penlidis: penlidis@uwaterloo.ca 


\section{GRAPHICAL ABSTRACT}

An intelligent optimizer capable of handling multi-objective optimizations was developed based on Artificial Intelligence techniques to recognize copolymer microstructural patterns applying heuristic search strategies and identify optimal copolymerization recipes for synthesizing predefined copolymers.

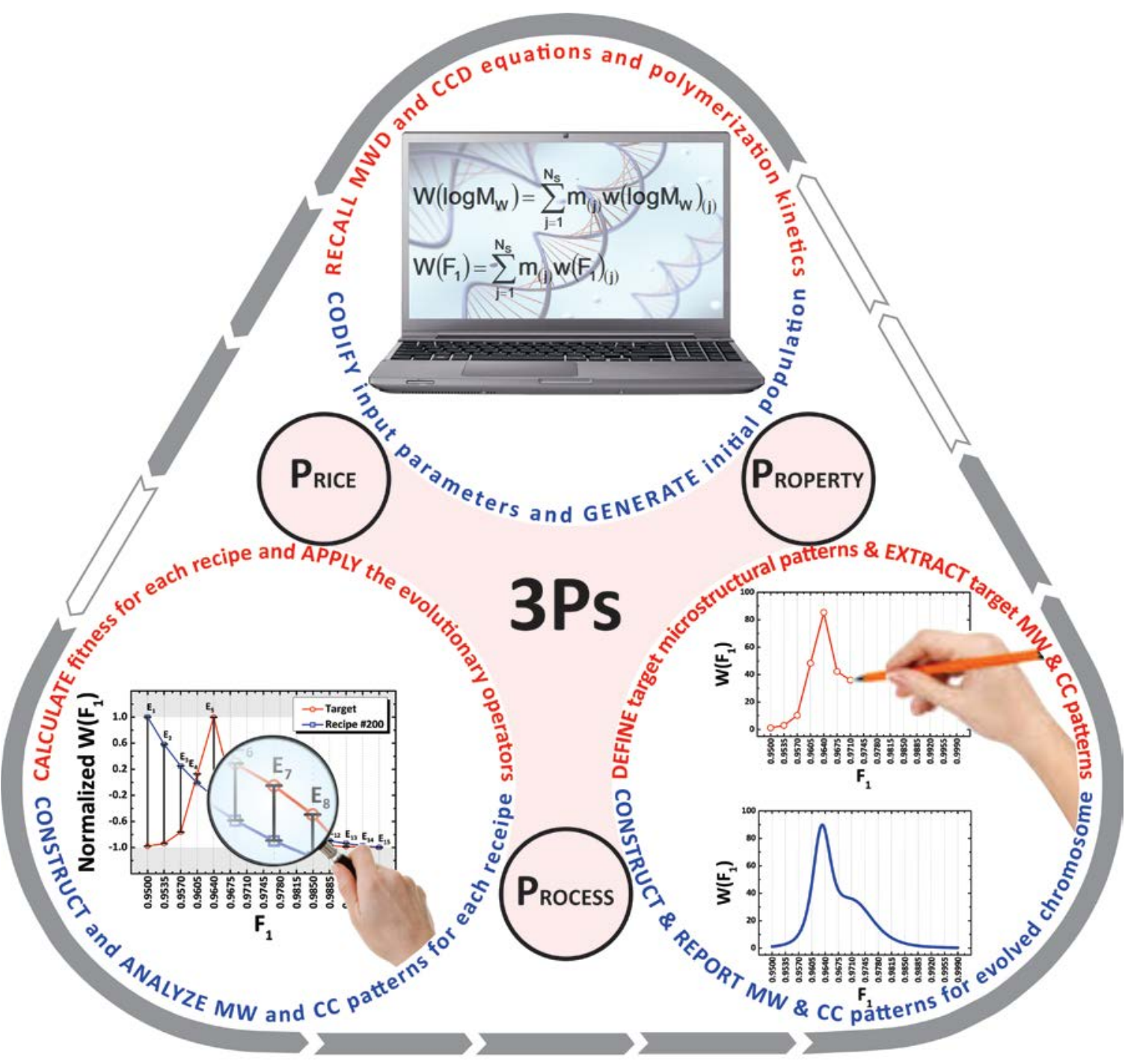




\section{ABSTRACT}

Manipulation and optimization of copolymer microstructure for tailoring final properties is of great importance in macromolecular science and engineering. Uncovering the complexities of the interrelationships between copolymerization recipe and copolymer microstructure (a challenging field of study in its own right) is a multi-objective optimization problem, which has attracted a lot of attention in the last 10-15 years. In the present study, a powerful optimizer was developed based on the Non-dominated Sorting Genetic Algorithm (NSGA-II) for transforming desired microstructural copolymerization profiles, including molecular weight distribution (MWD) and chemical composition distribution (CCD), back to optimal copolymerization recipes and operating conditions. The optimizer developed has the beneficial features of robust machine learning and multiobjective optimization based upon heuristic search strategies. The metallocene-catalyzed ethylene/ $\alpha$-olefin copolymerization was selected as a sufficiently complex system to challenge the proposed optimization tool. The developed computer code was used to explore copolymerization recipes (polymerization temperature and concentrations of ethylene, 1-butene, cocatalyst, and hydrogen) needed to synthesize copolymers having desired microstructural features. Based on the results obtained, it is now possible to produce various grades or tailor-make the copolymer structure by suggesting the 'best' copolymerization recipe/conditions as reliably as possible.

Keywords: Heuristic Search Strategy; Copolymerization; Microstructure; Multi-objective Optimization; Genetic Algorithms; Polyethylene 


\section{INTRODUCTION}

Manipulation and fine-tuning of copolymer properties has so far been realized mostly through detailed mathematical modeling for the simultaneous regulation of microstructural features of copolymer chains (monomer sequences/arrangements and chain length); see, for instance, [1-5]. Understanding the microstructure-property relationships in copolymerization is a rather cumbersome activity, and this arises mainly due to the complexity of the macromolecular reactions. It has been generally accepted that the molecular weight distribution (MWD) and the copolymer composition distribution (CCD) (and/or sequence length distribution, SLD) are the most important distributional properties representing the copolymer microstructure quantitatively $[6,7]$. Nevertheless, both the characterization and optimization of copolymer microstructure are still a challenging field of study in polymer science and engineering. This is mostly due to the fact that variations in MWD and CCD (SLD) patterns can be severely nonlinear. More importantly, there is a need for a robust and versatile tool capable of foreseeing optimal operational conditions resulting in desirable microstructural patterns, i.e., a way of backward tracking copolymerization recipes/operating conditions from desirable properties.

Basically, fine-tuning of copolymer microstructure and properties is intrinsically a multiobjective optimization problem and needs to be managed by a robust and powerful multiobjective optimization method. In contrast to time-consuming and often ineffective classical deterministic and stochastic approaches like random and/or exhaustive search strategies, more recent 'Computational Intelligence'-based optimization techniques have demonstrated significant benefits and outstanding capabilities in different optimization fields [8-10].

All Artificial Intelligence techniques share critical components of 'intelligence', including learning, generalization, and decision-making, for modeling and optimization of complex nonlinear problems. Artificial Neural Networks (ANNs) and Fuzzy Logic Systems are very powerful intelligent 'modelers', while the most popular heuristic search strategies 
(intelligent 'optimizers') include Swarm Intelligence, Simulated Annealing, Particle Swarm Optimization (PSO), and Genetic Algorithms (GAs) [11].

Generally speaking, ANNs are specifically designed as black-box modeling approaches to explore the nonlinear behavior of systems where mechanistic description of the interrelationships between input and output variables is unknown/overly complex [12]. Uses of ANNs in modeling polymerizations have been reported in several studies [13-19]. Although ANNs are essentially modeling techniques, they can also be applied in optimization of copolymer properties via a backward route. To do this, theoretical or experimental polymerization data collected for a well-defined number of polymerization scenarios (i.e., polymerization recipes and operating conditions as inputs and corresponding copolymer properties as outputs) are considered by a predefined ANN. Through training and testing stages, the parameters of the network, including weights and biases, are determined. The well-trained ANN is then capable of correlating inputs, i.e., copolymerization recipe/conditions, to copolymer properties as outputs. In fact, the ANN can predict the final copolymer properties for any given polymerization recipe. For the inverse route, i.e., when properties are set and polymerization conditions are required, another ANN has to be constructed and trained, which results in different sets of weights and biases, independent of those obtained via the forward route. Decisionmaking absolutely depends on the extent to which the real behavior of the system has been successfully imitated by the trained ANN.

In recent work [20], ANNs were applied for the optimization of ethylene/1-butene copolymerization with a two-single-site-type catalyst. Apparently, ethylene/ $\alpha$-olefin copolymers are appropriate case studies for exploring structure-property relationships, as a relatively broad range of microstructural patterns can be obtained depending on the catalyst composition and copolymerization conditions [21, 22]. Two ANNs (forward and inverse models) were trained and tested to find the interrelationship between inputs (i.e., copolymerization conditions including polymerization temperature and concentrations of ethylene, 1-butene, cocatalyst, and hydrogen as $\mathrm{X}$ variables) and outputs (i.e., microstructural patterns including MWD and CCD as Y responses) [20]. The forward model 
was responsible for predicting the $\mathrm{Y}$ responses for any given set of the $\mathrm{X}$ variables, while the inverse model was arranged to function in the opposite manner, i.e., to determine the polymerization conditions required to deliver a given polymer microstructure. However, an ANN is not necessarily the best choice of an 'expert' optimization technique. Although most of the Artificial Intelligence techniques can be interchangeably applied for both modeling and optimization, each one is principally designed and developed for either modeling or optimization purposes. Basically, ANNs are more preferable for modeling purposes. Secondly, constructing and training an ANN not only requires the collection of an adequate (usually large) amount of experimental or theoretical data but also it is essentially a computationally time-consuming process, especially in case of problems having a large number of inputs and/or outputs. In addition, the training and test datasets should be collected in a precise manner in order to be appropriately representative of the whole search space. Last but not least, ANN, being a black-box 'modeler', once trained, it returns only one output for any given input. On the other hand, multi-objective problems have multiple solutions in principle, known as Pareto optimal solutions.

A more powerful optimization tool is needed for the regulation of MWD and CCD/SLD. This tool should be capable of satisfying several predefined microstructural targets. Among all 'computationally intelligent' optimization methods and also classical deterministic and stochastic optimization techniques, the Non-dominated Sorting Genetic Algorithm (NSGA-II) is one of the most powerful methods to handle various multiobjective problems concurrently. Although NSGA-II has been successfully applied to manage multi-objective optimization problems in different fields of study, it has never been employed in the manipulation of copolymer microstructure [23-26].

In this work, a computer code was developed based on NSGA-II to optimize concurrently the MWD and CCD of ethylene/ $\alpha$-olefin copolymers in terms of polymerization temperature and concentrations of ethylene, 1-butene, cocatalyst, and hydrogen. In contrast to the ANN modeling approach, NSGA-II is an expert optimizer and has the capability of decision-making in case of complex multi-objective problems, hence it 
enables identification of all local optima and the global optimum [27-29]. The developed optimization algorithm was capable of precisely determining the copolymerization recipe and conditions required for the production of copolymers with preset desirable MWD and CCD patterns.

\section{MODEL DEVELOPMENT}

Mathematical modeling of metallocene-based copolymerization of ethylene with $\alpha$ olefins containing multi-site catalytic systems has been comprehensively dealt with [2]. Macroscopic properties are governed by distributions of short-chain branches and molecular weights. Two equations were used for tracking microstructural changes involving MWD and CCD, as follows:

$$
\begin{aligned}
& W\left(\log M_{W}\right)=\sum_{j=1}^{N_{S}} m_{(j)} w\left(\log M_{W}\right)_{(j)} \\
& W\left(F_{1}\right)=\sum_{j=1}^{N_{S}} m_{(j)} w\left(F_{1}\right)_{(j)}
\end{aligned}
$$

In the above summation equations, the subscript $j$ denotes the site type, $m_{(j)}$ is the mass fraction of polymer produced on site type $j, M_{w}$ refers to molecular weight, $w(-)_{(j)}$ denotes the weight fraction (distribution) of molecular weight and/or chemical composition of polymer produced on site type $j, W(-)$ denotes the total weight (distribution) of molecular weight and/or chemical composition, $N_{s}$ is the total number of active site types on the catalyst, and $F_{1}$ is the molar fraction of ethylene monomer in the copolymer. More information on the derivation of these equations can be found elsewhere $[2,20]$. Despite the fact that the above equations are capable of precisely predicting the variations of MWD and CCD for a wide range of operational conditions (moving from $X$ to $Y$ ), the establishment of structure-property relationships in such systems needs continuous update due to the fact that the interrelationships between copolymerization conditions and microstructural patterns are severely nonlinear (moving in an inverse manner, i.e., 
from $\mathrm{Y}$ to $\mathrm{X}$ ). For instance, a small change in copolymerization recipe/conditions may cause changing MWD and/or CCD profiles from unimodal to bimodal, and vice versa. More importantly, the problem becomes even more complex and multi-dimensional when one needs to find optimal polymerization conditions necessary for production of copolymers with desired microstructures. Therefore, considering the nonlinearity of the problem, copolymer microstructure manipulation and regulation applying the above summation equations is intrinsically a multi-objective optimization problem with multiplicity of solutions for any predefined copolymer microstructure.

Basically, all typical problems are multi-objective. Usually, single-objective problems are mostly defined for the sake of simplicity for illustration purposes. This means that (i) most of the time one just selects/considers the most important objective and neglects the others to convert multi-objective problems into single-objective ones; or, (ii) sometimes one just selects one objective and considers one or more other objectives as constraints. In both cases, the optimization procedures are simplified and redefined as singleobjective optimizations. The fact is that the best solution for a multi-objective problem is obtained only if an expert multi-objective optimization method is applied.

Multi-objective optimization problems, like the simultaneous optimization of MWD and CCD can be handled by both classical and evolutionary algorithms. Classical algorithms, such as weighted sum, goal programming, goal attainment, and $\varepsilon$-constraint, are mostly based on deterministic transition rules and attempt to 'scalarize' iteratively multiple objectives in exploring a set of Pareto optimal solutions [23]. Also, classical approaches mostly convert a multi-objective problem into a simplified single-objective optimization problem with or without considering certain constraints. By contrast, evolutionary algorithms use stochastic principles to find solutions in a single simulation run [24]. Principally, in solving a multi-objective problem, difficulties may arise from the execution of the searching and decision-making phases of optimization. This requires development of sophisticated computer codes and hybridization of computational algorithms in order to find the best optimal conditions and subsequently warrant multi-variable optimization of two or more targets $[25,26]$. 
Nowadays, Genetic Algorithms combined with heuristic search strategies are the most popular optimization techniques, widely applied in different fields of study considering their flexibility and ability to handle a large variety of problems. They are based on generating a population of potential solutions and stochastically evolving them toward better solutions via the application of powerful genetic operators. Not only are Genetic Algorithms masterful in single-objective optimizations, but also capable of handling multiobjective optimizations with two or more objectives and constraints concurrently. Among different Genetic Algorithms, NSGA-II is a unique multi-objective version of the family established primarily based on the domination concept. Undoubtedly, it can be considered as one of the most applied optimization techniques in different fields of science and technology [23-26].

Due to these reasons, NSGA-II is selected and applied for recognition and multi-objective optimization of MWD and CCD patterns in a metallocene-catalyzed copolymerization of ethylene with an $\alpha$-olefin. The ethylene/1-butene system with two-single-site-type catalyst is selected as a case study. The reaction scheme and kinetic parameters used are as described in [20]. The proposed copolymerization mechanism consists of site activation, chain initiation, propagation, chain transfer to monomer, chain transfer to hydrogen, chain transfer to cocatalyst, $\beta$-hydride elimination, and catalyst deactivation. The kinetic constants for the copolymerization of ethylene with 1-butene at $360 \mathrm{~K}$ are given in Table 1, as cited in [20]. 
Table 1. Kinetics constants and activation energies for ethylene/1-butene copolymerization at $360 \mathrm{~K}[20]$.

\begin{tabular}{|c|c|c|c|c|c|c|}
\hline Mechanism & $\begin{array}{l}\text { Kinetic } \\
\text { constant }\end{array}$ & Units & Site 1 & Site 2 & $\begin{array}{l}\text { Activation energy } \\
\text { Site } 1\left(\text { cal.mol }{ }^{-1}\right)\end{array}$ & $\begin{array}{l}\text { Activation energy } \\
\text { Site } 2 \text { (cal.mol }{ }^{-1} \text { ) }\end{array}$ \\
\hline Activation & $k_{\mathrm{f}}$ & $\mathrm{L} \mathrm{mole}^{-1} \mathrm{~s}^{-1}$ & 1 & 1 & 12945.44 & 12946.44 \\
\hline \multirow[t]{2}{*}{ Initiation } & $k_{i, 1}$ & $\mathrm{~L} \mathrm{~mole}^{-1} \mathrm{~s}^{-1}$ & 1 & 1 & 9000 & 9100 \\
\hline & $k_{\mathrm{i}, 2}$ & $\mathrm{~L} \mathrm{~mole}^{-1} \mathrm{~s}^{-1}$ & 0.14 & 0.14 & 9200 & 9300 \\
\hline \multirow[t]{4}{*}{ Propagation } & $k_{\mathrm{p}, 11}$ & $\mathrm{~L} \mathrm{~mole}^{-1} \mathrm{~s}^{-1}$ & 8.5 & 8.5 & 10000 & 10100 \\
\hline & $k_{\mathrm{p}, 12}$ & $\mathrm{~L} \mathrm{~mole}^{-1} \mathrm{~s}^{-1}$ & 2 & 1.5 & 15001 & 15101 \\
\hline & $k_{\mathrm{p}, 21}$ & $\mathrm{~L} \mathrm{~mole}^{-1} \mathrm{~s}^{-1}$ & 6.4 & 6.4 & 10001 & 10101 \\
\hline & $k_{\mathrm{p}, 22}$ & $\mathrm{~L} \mathrm{~mole}^{-1} \mathrm{~s}^{-1}$ & 1.5 & 2.26 & 15000 & 15100 \\
\hline Transfer to & $k_{\mathrm{M}, 11}$ & $\mathrm{~L} \mathrm{~mole}^{-1} \mathrm{~s}^{-1}$ & 0.0021 & 0.0021 & 19824.21 & 19834 \\
\hline \multirow[t]{3}{*}{ Monomer } & $k_{\mathrm{M}, 12}$ & $\mathrm{~L} \mathrm{~mole}^{-1} \mathrm{~s}^{-1}$ & 0.006 & 0.11 & 19825.21 & 19835 \\
\hline & $k_{\mathrm{M}, 21}$ & $\mathrm{~L} \mathrm{~mole}^{-1} \mathrm{~s}^{-1}$ & 0.0021 & 0.0021 & 19826.21 & 19836 \\
\hline & $k_{\mathrm{M}, 22}$ & $\mathrm{~L} \mathrm{~mole}^{-1} \mathrm{~s}^{-1}$ & 0.006 & 0.11 & 19827.21 & 19837 \\
\hline \multirow[t]{2}{*}{ Transfer to $\mathrm{H}_{2}$} & $k_{\mathrm{H}, 1}$ & $\mathrm{~L} \mathrm{~mole}^{-1} \mathrm{~s}^{-1}$ & 0.088 & 0.37 & 19820.21 & 19830 \\
\hline & $k_{\mathrm{H}, 2}$ & $\mathrm{~L} \mathrm{~mole}^{-1} \mathrm{~s}^{-1}$ & 0.088 & 0.37 & 19821.21 & 19831 \\
\hline Transfer to & $k_{\mathrm{A}, 1}$ & $\mathrm{~L} \mathrm{~mole}^{-1} \mathrm{~s}^{-1}$ & 0.024 & 0.12 & 19822.21 & 19832 \\
\hline cocatalyst & $k_{\mathrm{A}, 2}$ & $\mathrm{~L} \mathrm{~mole}^{-1} \mathrm{~s}^{-1}$ & 0.048 & 0.24 & 19823.21 & 19833 \\
\hline$\beta$-Hydride & $k_{\beta, 1}$ & $\mathrm{~s}^{-1}$ & 0.0001 & 0.0001 & 15005 & 15006 \\
\hline elimination & $k_{\beta, 2}$ & $s^{-1}$ & 0.0001 & 0.0001 & 15008 & 15007 \\
\hline Deactivation & $k_{\mathrm{d}}$ & $\mathrm{s}^{-1}$ & 0.0001 & 0.0001 & 12900 & 13500 \\
\hline
\end{tabular}

Possible ranges of variation of copolymerization recipe/conditions used in the multiobjective optimization of MWD and CCD patterns are presented in Table 2, again as implemented in [20].

Table 2. Range of variation of copolymerization conditions (as per [20]) used in multiobjective optimizations.

\begin{tabular}{|c|c|c|c|}
\hline \multirow{2}{*}{$\begin{array}{l}\text { Operation condition } \\
\text { Ethylene }\end{array}$} & \multirow{2}{*}{$\begin{array}{l}\text { Unit } \\
\mathrm{mol} \mathrm{L}^{-1}\end{array}$} & \multicolumn{2}{|c|}{ Range of Values } \\
\hline & & 0.200 & - $\quad 3.740$ \\
\hline 1-Butene & $\mathrm{mol} \mathrm{L}^{-1}$ & 0.030 & - $\quad 1.050$ \\
\hline Hydrogen & $\mathrm{mol} \mathrm{L}^{-1}$ & 0.0001 & - $\quad 0.0100$ \\
\hline Co-catalyst & $\mathrm{mol} \mathrm{L}^{-1}$ & 0.0003 & - 0.0143 \\
\hline Temperature & ${ }^{\circ} \mathrm{C}$ & 70.0 & - 90.0 \\
\hline Average reactor residence time $\left(t_{r}\right)$ & $\mathrm{s}$ & \multicolumn{2}{|l|}{1000} \\
\hline The total molar flow rate of catalyst precursor $\left(C^{\mathrm{in}}\right)$ & mole s$^{-1}$ & \multicolumn{2}{|l|}{0.00001} \\
\hline Molar fraction of site type $\mathrm{j}\left(x_{(j)}\right)$ & - & \multicolumn{2}{|c|}{$0.5(\mathrm{j}=1$ and 2$)$} \\
\hline
\end{tabular}

Four different microstructural patterns, referred to from now on as Cases I-IV, are defined as target ethylene/1-butene copolymer microstructures to be imitated, patterned, and 
optimized by NSGA-II. To do this, an in-house computer code was written in PASCAL programming language (Lazarus IDE 1.6.4) and compiled into 64-bits executable using FPC 2.6.2. The program was run on a desktop computer with Intel Core i7-3770K $(3.50 \mathrm{GHz})$, $32 \mathrm{~GB}$ of memory (2133 MHz), under Windows 7 Ultimate 64-bit operating system. The optimization runtime took less than two minutes for all cases studied in this work (Case I: 17 s, Case II: 112 s, Case III: 83 s, and Case IV: 98 s).

The developed code is capable of tracking the copolymer microstructural patterns as a function of copolymerization recipe/conditions, i.e., solving multi-objective optimization problems (e.g., arrive at the appropriate copolymerization recipes to synthesize copolymers with preset MWD and CCD profiles).

Scheme 1 is a graphical flowchart that demonstrates the multi-objective optimization algorithm developed in this work. First of all, the copolymerization recipe/conditions (reaction temperature $(T)$, concentrations of ethylene $(E), 1$-butene $(B)$, cocatalyst $(C)$, and hydrogen $(H))$ are codified into a chromosome-like structure with tightly connected genes resembling a potential solution for the problem under study (Scheme 2). In this chromosome, 30 targets are considered to represent the patterns of MWD and CCD, responsible for transferring microstructural information. In other words, both MWD and CCD profiles are divided into 14 identical intervals in an attempt to specify 15 discretized data points as representatives of MWD and CCD patterns. The developed computer code identifies chromosomes of the same structure, but with different genes. The computer program is capable of receiving a coded copolymerization recipe as a genotype and decoding it via the application of the previously described summation equations into the corresponding phenotype, i.e., MWD and CCD patterns. As can be observed, NSGA-II is able to make a direct interconnection between genotypes and phenotype. In other words, it receives copolymerization recipes and calculates/visualizes MWD and CCD patterns for each recipe via recalling online the summation equations (1) and (2). In contrast, ANN recalls the summation equations in an offline mode, i.e., as an 'avatar' for the summation equations; it can only estimate equations (1) and (2). Hence, there inevitably exists an intrinsic error in the prediction of microstructural patterns when ANNs are utilized for 
multi-objective optimization purposes. Also, changing the potential operating ranges for copolymerization recipes/conditions, NSGA-Il can still handle the optimization of microstructural patterns in the operating space, whereas new ANNs should be constructed and trained for the new search space, every time the operating space changes.

Secondly, the predefined target copolymer with preset MWD and CCD patterns is directly received by the computer code. As per previous work in the literature, 15 data points on both MWD and CCD microstructural patterns are specified by equally-spaced slicing of input patterns, which is shown to be adequate for reflecting the behavior of such distribution curves [20]. Then, in the third step, an initial population of chromosomes (i.e., copolymerization recipes) is generated randomly. It is worth mentioning that in all Genetic Algorithms the population generation is random only in the first iteration. In other words, new generations emerge via the genetic operators capable of adjusting the gene(s) values to evolve the population and produce optimum solutions. In the fourth step, the optimization algorithm calculates/visualizes the MWD and CCD patterns for the generated chromosomes one by one recalling the summation equations (equations (1) and (2)). Since the summation equations are directly recalled by NSGA-II instead of being approximated by the trained ANN (as in [20]), the degree of accuracy is much higher.

In the fifth step, the visualized MWD and CCD patterns of each chromosome are separately compared with the target MWD and CCD patterns to evaluate the fitness via determination of their deviation from the predefined targets. The calculated errors are reported as the fitness values of each chromosome. Obviously, for each chromosome, two error values represent the amount of deviation from the target MWD and CCD patterns, respectively. 


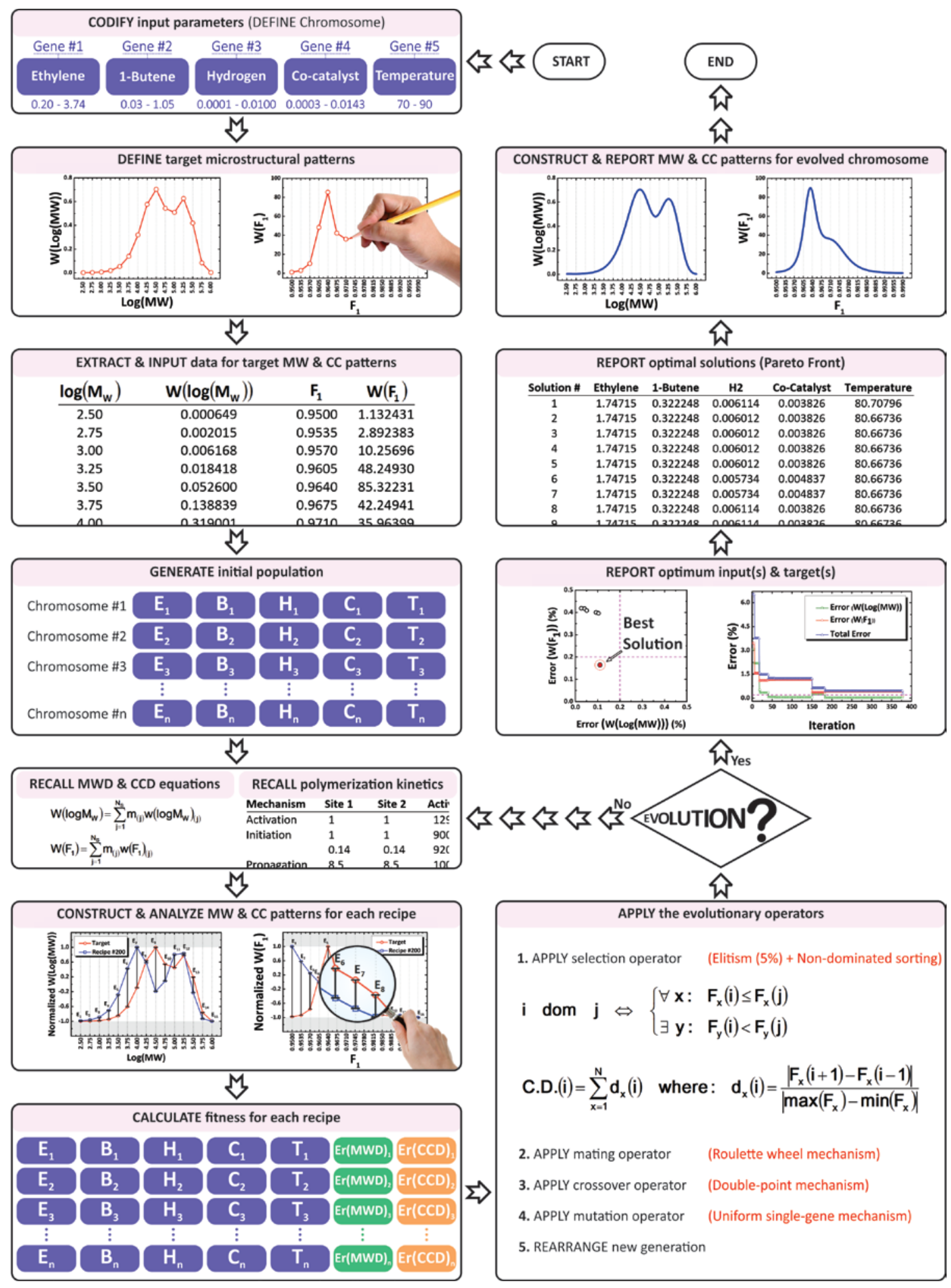

Scheme 1. Detailed graphical flowchart based on NSGA-II for copolymer microstructure recognition and optimization. 
The genetic algorithm includes selection, sorting, mating, crossover, and mutation operators, and these are applied in the sixth step to establish new generations via an evolutionary pathway. Basically, the main difference between NSGA-II and other singleobjective versions of Genetic Algorithms is the mechanism of sorting the potential solutions. NSGA-II utilizes the domination concept to sort chromosomes in a multiobjective optimization framework. According to this mechanism, the chromosomes are sorted based on the "quality" and "diversity" of the solutions. The former criterion organizes the solutions into classes named Pareto fronts, whereas the latter separately puts the members of each Pareto front into order by fitness values.

\begin{tabular}{|c|c|c|c|c|}
\hline \multicolumn{5}{|c|}{ Chromosome \#j (Recipe \#j) } \\
\hline Gene \#1 & Gene \#2 & Gene \#3 & Gene \#4 & Gene \#5 \\
\hline Ethylene & 1-Butene & Hydrogen & Co-catalyst & Temperature \\
\hline $0.20 \cdot 3.74$ & $0.03-1.05$ & $0.0001 \cdot 0.0100$ & $0.0003-0.0143$ & $70-90$ \\
\hline
\end{tabular}

Molecular Weight Distribution Copolymer Composition Distribution

\begin{tabular}{|c|c|c|c|}
\hline Target \#1 & Target \#15 & Target \#16 & Target \#30 \\
\hline$\left.\left(M_{w}\right)\right)_{1}$ & $W\left(\log \left(M_{w}\right)\right)_{15}$ & $W\left(F_{1}\right)_{1}$ & $W\left(F_{1}\right)_{15}$ \\
\hline $\log \left(\mathrm{M}_{\mathrm{w}}\right)=2.50$ & $\log \left(M_{w}\right)=6.00$ & $F_{1}=0.9500$ & $F_{1}=0.9990$ \\
\hline
\end{tabular}

Scheme 2. Defined chromosome-like structure illustrating (i) the variation interval for each polymerization variable and (ii) the microstructural patterns with 15 equallyspaced MWD and CCD target points to be optimized.

To classify the chromosomes based on the quality of solutions, the domination concept is applied. A certain chromosome (e.g., chromosome i) dominates (over) another chromosome (e.g., chromosome $j$ ) if it is not worse than the latter chromosome in all predefined objectives and definitely better in at least one objective (see Equation 3). If all objectives should be mutually minimized, then the domination concept is expressed as the following mathematical equation:

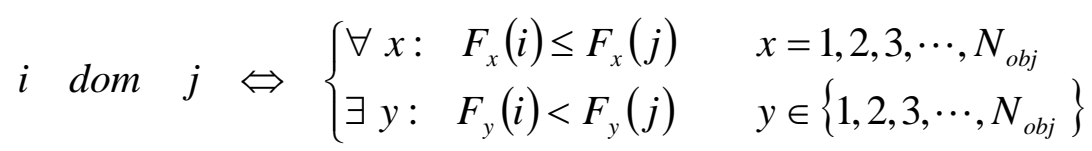

where $F_{x}(i)$ is the fitness value of chromosome $i$ in objective $x$ and $N_{o b j}$ is the total number of predefined objectives. Comparing all possible pairs of solutions, a number or rank is assigned to each chromosome based on the dominations. The chromosomes are 
subsequently organized into a set of Pareto fronts. Accordingly, non-dominated chromosomes are placed in the first Pareto front, while the second Pareto front hosts those chromosomes dominated once by the members in the first front and the front classification goes on. Afterwards, the chromosomes in the first front are given a rank value of 1 , those in the second front are assigned the rank value of 2 , and so on.

Finally, the chromosomes in each Pareto front are separately sorted based upon the Crowding Distance (C.D.) criterion, defined as follows:

C.D. $(i)=\sum_{x=1}^{N_{\text {obj }}} d_{x}(i) \quad$ where $: \quad d_{x}(i)=\frac{\left|F_{x}(i+1)-F_{x}(i-1)\right|}{\left|\max \left(F_{x}\right)-\min \left(F_{x}\right)\right|}$

In this equation, $d_{x}(i)$ is the crowding distance of chromosome $i$ with respect to objective $x$. Also, $\min \left(F_{x}\right)$ and $\max \left(F_{x}\right)$ are the minimum and maximum values of objective $x$, respectively. $N_{o b j}$ denotes the number of objectives and CD $(i)$ is the crowding distance of chromosome $i$.

After sorting and selecting the most suitable chromosomes, these chromosomes are utilized to generate new members to be replaced by rejected chromosomes of the previous generation. This is handled by two well-known powerful intelligent genetic operators; crossover and mutation. Both operators are expert stochastic search tools but their searching mechanism and implementation are quite different. Crossover is mainly applied for exploitation, while mutation is employed for exploration. More specifically, the crossover operator takes two chromosomes as parents from the existing population and attempts to generate two new chromosomes as offsprings which are more similar to the parents. Hence, the crossover operator seeks promising regions in the hope to find additional solutions, i.e., local optima. On the other hand, mutation influences a single chromosome and changes it into a new chromosome which may or may not be in the current population. Thus, the mutation operator seeks the unexplored regions to guarantee that all regions of the search space are thoroughly explored and the search is 
not confined to limited regions. It is clear that the rate and type of crossover and mutation can be regulated depending on the problem under study.

The evolutionary process stops whenever one or more evolved solutions satisfy the predefined target(s). Then, optimal solution(s) containing polymerization temperature and concentrations of the specific ingredients are reported. Lastly, the MWD and CCD patterns are calculated at predicted optimum polymerization conditions using the summation equations in order to evaluate the authenticity of the optimization process.

\section{RESULTS AND DISCUSSION}

\subsection{Verification of NSGA-II performance}

Two different copolymerization recipes (Cases I and II) are shown in Table 3. Their corresponding MWD and CCD patterns are theoretically calculated/visualized applying summation equations 1 and 2 considering the copolymerization reaction mechanism and kinetic parameters given in Tables 1 and 2 (blue curves at the bottom plots of Table 3). Then, as mentioned in the previous section, both MWD and CCD profiles are divided into 14 identical intervals to specify 15 discretized data points as representative of the MWD and CCD patterns of the target copolymers, respectively (red lines at the bottom plots of Table 3). It can be observed that the target MWD and CCD patterns (red curves) yielded by connecting neighboring pairs of target points by a straight line can appropriately represent the theoretical MWD and CCD patterns. In order to demonstrate the capabilities of the NSGA-II code written in this work, the MWD patterns of Case I and Case II are deliberately taken in a manner to exhibit a bimodal and a unimodal distribution, respectively. The assigned points are chosen at specified steps of $\log (\mathrm{MW})$ and $F_{1}$, represented by hollow circles in the plots at the bottom of Table 3 . The values corresponding to each point are explicitly cited in Table 3 for Cases I and II.

Now, for both cases, the aforementioned target MWD and CCD patterns are simultaneously fed into the NSGA-II optimization code to assess the ability of the developed code with respect to recognizing MWD and CCD patterns and prediction of optimum copolymerization recipe(s) (i.e., those recipies defined in this section to produce 
Cases I and II). As mentioned in the model development section, the NSGA-II algorithm randomly produces a wide variety of recipes as the initial population in the first step in an attempt to recognize the microstructural patterns it has received as model inputs. In the current study, the initial population size is set to be 500 recipes. In the second step, the algorithm recalls summation equations (1) and (2) for calculating/visualizing MWD and CCD patterns of each randomly generated recipe. The obtained microstructural patterns are utilized to evaluate the error values in prediction of $\log (\mathrm{MW})$ and $\mathrm{F}_{1}$ with respect to the predefined targets. It is obvious that except for the initial population, NSGA-II generates the next populations intelligently via the evolutionary manner described in section 2 .

Table 3. Polymerization conditions along with corresponding target MWD and CCD patterns used in multi-objective optimization of copolymer microstructure. 


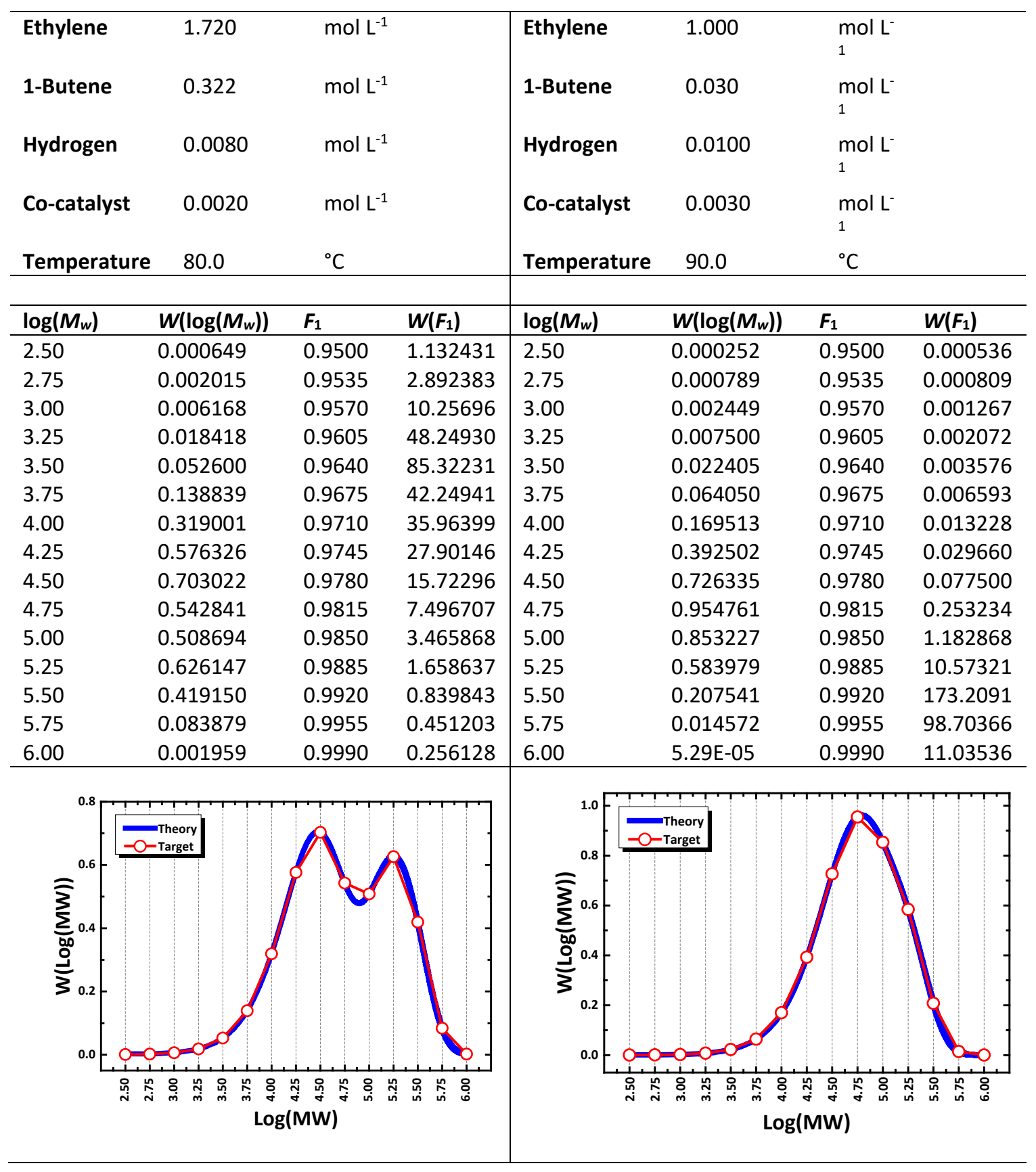



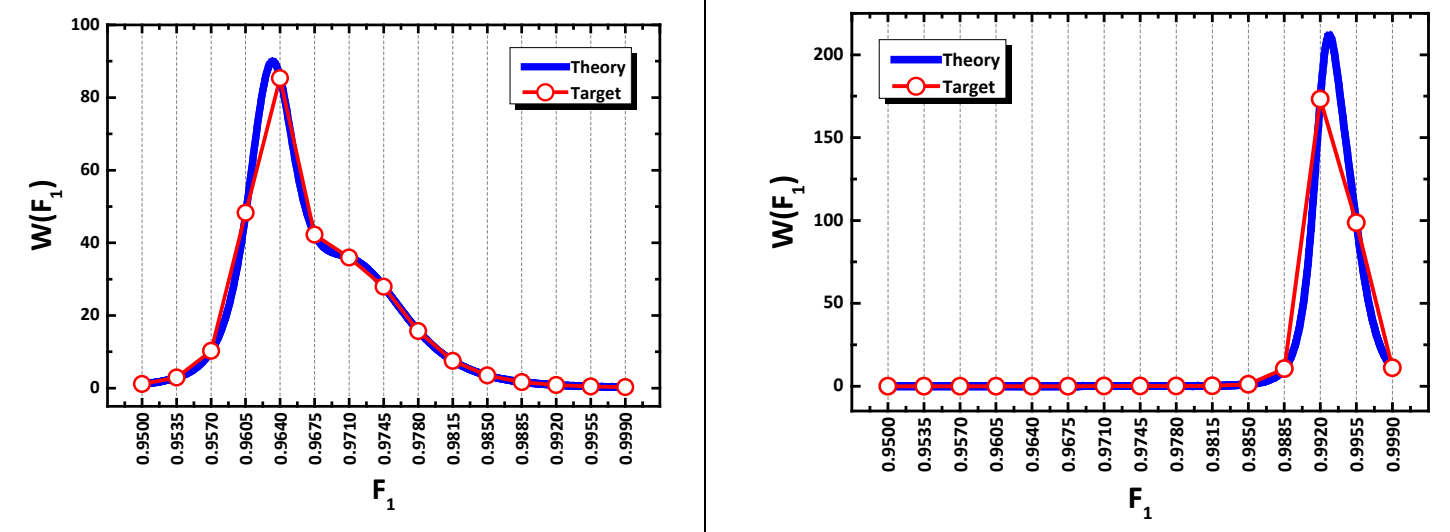

The error values calculated for a given recipe are taken as the fitness values for that specific recipe. The roulette wheel mechanism, double-point mechanism, and uniform single-gene mechanism are utilized for mating, crossover, and mutation operators, respectively. As already mentioned earlier, one of the beneficial aspects of NSGA-II in optimization of microstructural patterns is its capability to recall directly the summation equations (equations (1) and (2)) in order to calculate the fitness values of the chromosomes. On the other hand, ANNs just imitate the behavior of the summation equations with an error which is normally preset in the training and testing stages. Put simply, the ANN approach utilizes the 'modeled' version of the summation equations with a built-in error.

Figure 1 provides a perspective on the evolutionary nature of the NSGA-II optimization code in which MWD and CCD patterns of a copolymer calculated by NSGA-II are plotted as a sample for recipe \#200 after 100 iterations. As can be observed, the algorithm compares the obtained microstructural patterns with the target patterns defined as Case I and calculates the corresponding errors. It should be emphasized that the maximum allowable error values, i.e., stopping condition in identifying target copolymer I, are arbitrarily set to be $0.2 \%$ for both MWD and CCD patterns. 
(A)

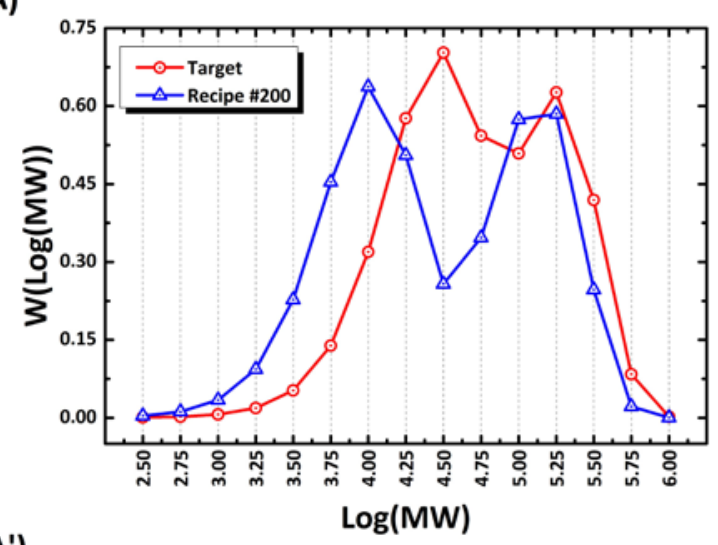

(A')

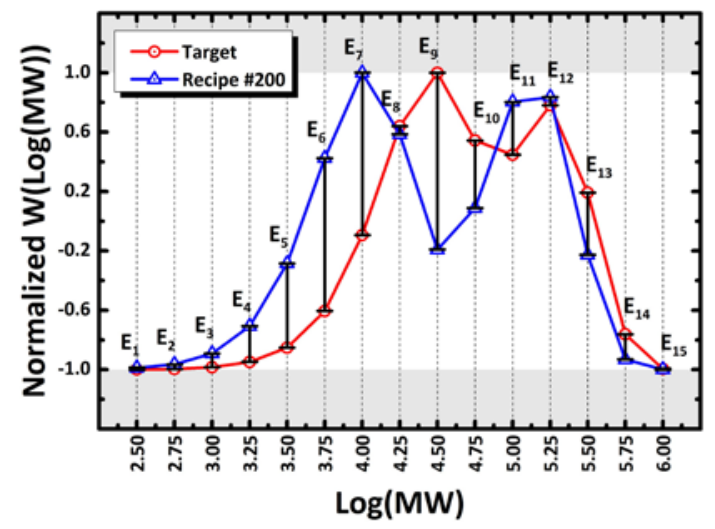

(B)

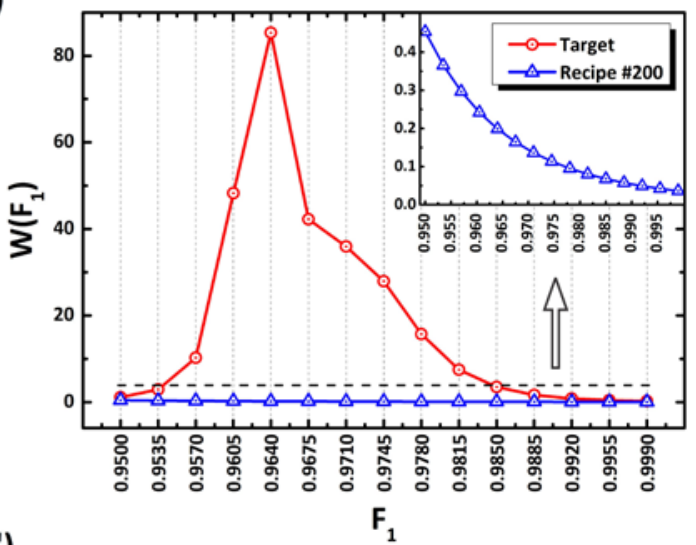

(B')

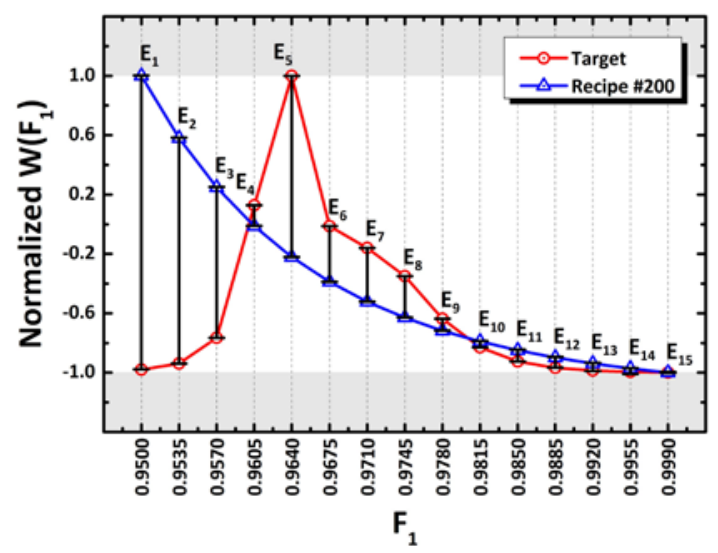

Figure 1. ( $A$ and $B$ ) MWD and CCD patterns of Recipe \#200 proposed by the optimization algorithm for Case I after 100 iterations; ( $A^{\prime}$ and $B^{\prime}$ ) Error calculation mechanism along with corresponding errors in prediction/recognition of the microstructural patterns for the same recipe and at the same epoch.

It can be observed that some local errors at target points located on the MWD and CCD curves (i.e., $E_{1}, E_{2} \ldots E_{15}$ specified in Figures $1 A^{\prime}$ and $1 B^{\prime}$ ) may have approached zero, but overall the NSGA-II algorithm needs to seek out and match target microstructural patterns by further interactions. Obviously, to make an appropriate comparison, both generated patterns and target patterns are separately normalized between -1 and +1 . The errors are then calculated through the summation of local errors for both MWD and CCD patterns. Figure 2 shows the results of the multi-objective optimization for the target copolymer defined in Case I, accomplished after 378 iterations. Interestingly, 18 solutions have successfully met the primary criterion of NSGA-II, i.e., the ranking criterion, but only one 
solution has been labeled as the best solution (Solution \#1), which satisfied the allowable error constraints, that is $0.2 \%$ for either MWD or CCD. Once the best solution was identified, the code stopped running. The other 17 solutions were those positioned in the first Pareto front as non-dominating solutions, whose errors in MWD and/or CCD exceeded $0.2 \%$. The corresponding microstructures were close to the one reported as the optimum solution (solution \#1). It is apparent from Figure 2 that the NSGA-II optimizer has successfully and simultaneously recognized the target patterns (both MWD and CCD patterns) and identified the optimum recipe for synthesizing the target copolymer in Case I.

(A)

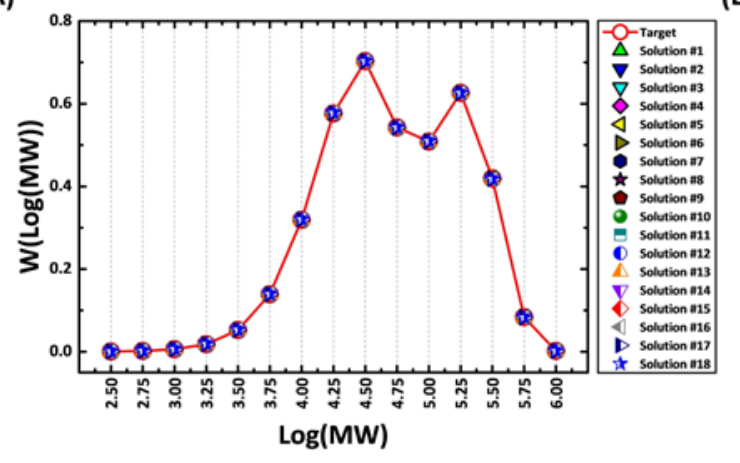

(B)

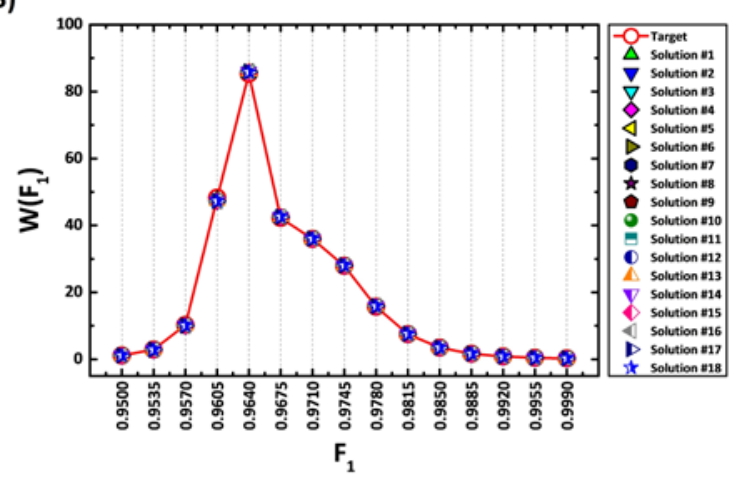

Figure 2. MWD (A) and CCD (B) patterns of target copolymer defined in Case I, achieved after 378 iterations.

The Pareto front and iteration errors in predicting the MWD and CCD patterns computed and reported by the NSGA-II code for target copolymer I are presented in Figure 3. It can be observed that the developed code can appropriately find the best solution in view of the $0.2 \%$ error (filled circle in Figure $3 \mathrm{~A}$ ). It is worth mentioning that there are some solutions (unfilled circles in Figure 3A) that only meet the allowable error of $0.2 \%$ with respect to the MWD pattern. These solutions have been distinguished by the NSGA-II code and bypassed as optimal solutions, because of the CCD error constraint not having been met, although they are all placed in the first Pareto front. The descending trends in the individual errors in MWD, CCD, and the total error during the optimization process of the 
target copolymer I emphasize further the reliability of the proposed optimization method (Figure 3B). At the final iteration, the exact error values were $0.1105,0.1639$, and 0.2744 $\%$, respectively.
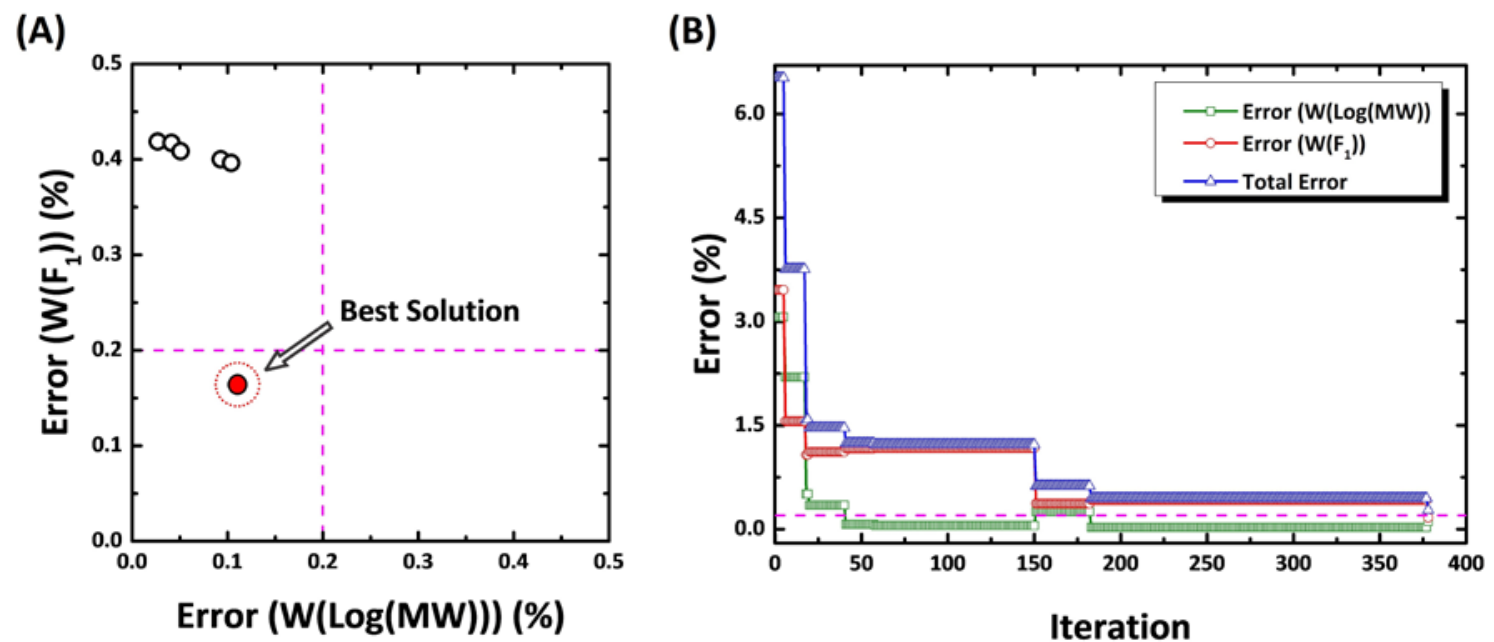

Figure 3. The Pareto optimal front $(A)$ and errors in prediction of MWD and $C C D$ patterns (B) proposed by the NSGA-II code for Case I target copolymer.

Figure 4 compares the microstructural patterns of the best solution (solution \#1) proposed by the optimizer (green symbols) with the theoretical patterns yielded from summation equations 1 and 2 (blue curves) and those considered by the code as the target copolymer I (red lines). It is obvious that NSGA-II has predicted the MWD and CCD profiles of the target copolymer I satisfactorily. The developed optimization code successfully recognized the MWD and CCD patterns simultaneously through a multiobjective optimization pathway. There is also evidence that the selected points are adequate and have an acceptable resolution to represent the real picture of the microstructural patterns in the case of target copolymer I. 

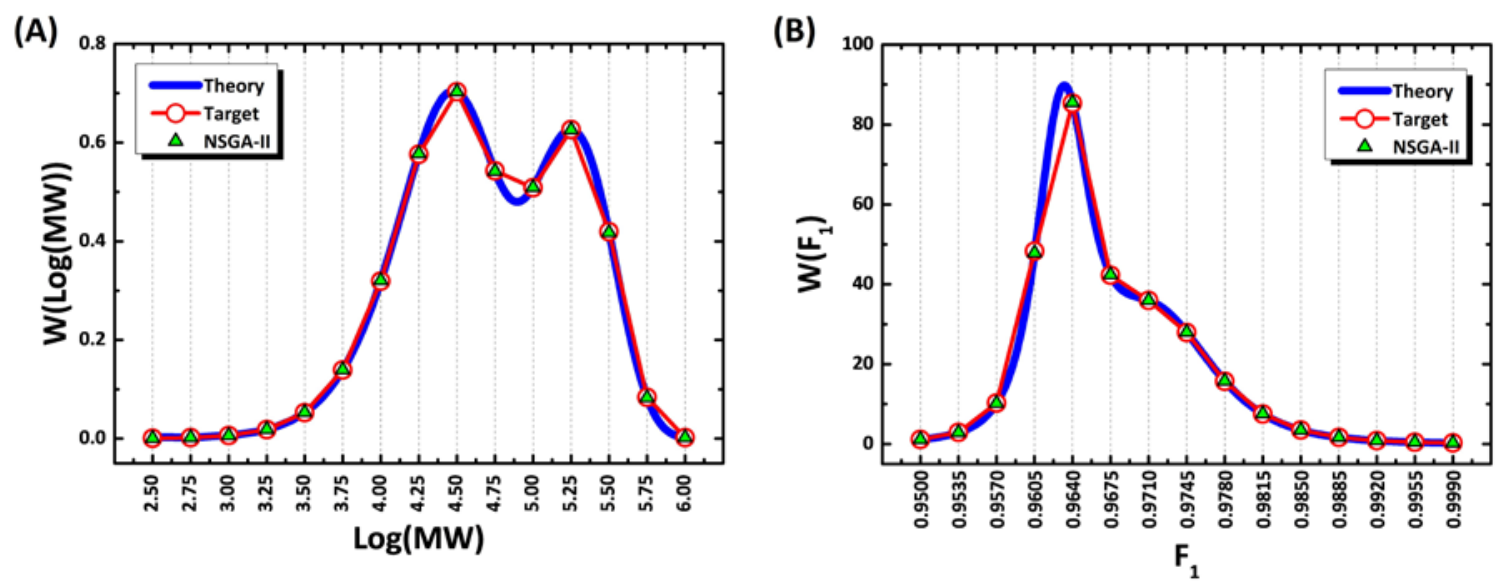

Figure 4. Comparison of the optimization results (NSGA-II) and predefined targets (theoretical plots obtained using summation equations 1 and 2 ) in case of copolymer I; MWD (A) and CCD (B) patterns.

Table 4 provides detailed information on the 18 solutions shown in Figure 2 and proposed by the developed NSGA-II code. These solutions are obtained at the 378th iteration, where only the first solution met the overall error criteria of $0.2 \%$ for both $\log (\mathrm{MW})$ and $F_{1}$ constraints. The others were separated and stored because of being non-dominating but located in the first Pareto front. It is apparent that all solutions are successful in identifying and reporting the best values for the target MWD and CCD patterns. The first column of Table 4 represents the solution number sorted in accordance with total error values from smaller to larger. The second to sixth columns represent the copolymerization recipes proposed by the NSGA-II optimizer, i.e., values of the operating variables including concentrations of ethylene, 1-butene, hydrogen, and cocatalyst, and the polymerization temperature. The seventh and eighth columns give errors in the optimization patterns of MWD and CCD, respectively. In the ninth and tenth columns are the values of the first and second criteria used in the multi-objective optimization of the MWD and CCD profiles, respectively. The former, named "Ranking", is calculated and assigned based on the sorting of solutions in view of the domination concept, which classifies solutions in different Pareto fronts. It can be seen that from the perspective of this first criterion all solutions are acceptable because of having been located in the first Pareto front. The 
latter, i.e., C.D. column, is indicative of the crowding distance of solutions located in each Pareto front. This second criterion is able to sort the solutions isolated in different Pareto fronts separately. The solutions scoring higher crowding distance values are more acceptable.

Table 4. Details of Pareto optimal solutions obtained at the last iteration for target copolymer I by NSGA-II optimizer.

\begin{tabular}{llllllllll}
\hline Solution & $\begin{array}{l}\text { Ethylene } \\
\left(\mathbf{m o l ~ L}^{-1}\right)\end{array}$ & $\begin{array}{l}\text { 1-Butene } \\
\left(\mathbf{m o l ~ L}^{-1}\right)\end{array}$ & $\begin{array}{l}\text { Hydrogen } \\
\left(\mathbf{m o l ~ L}^{-1}\right)\end{array}$ & $\begin{array}{l}\text { Cocatalyst } \\
\left(\mathbf{m o l ~ L}^{-1}\right)\end{array}$ & $\begin{array}{l}\text { Temperature } \\
\left({ }^{\circ} \mathbf{C}\right)\end{array}$ & $\begin{array}{l}\text { Error in MW } \\
(\%)\end{array}$ & $\begin{array}{l}\text { Error in } \mathbf{F}_{\mathbf{1}} \\
(\%)\end{array}$ & Ranking & C.D. \\
\hline 1 & 1.7212 & 0.3222 & 0.0081 & 0.0020 & 80.0080 & 0.1105 & 0.1639 & 1 & Infinity \\
2 & 1.7472 & 0.3222 & 0.0060 & 0.0038 & 80.6674 & 0.0268 & 0.4185 & 1 & Infinity \\
3 & 1.7472 & 0.3222 & 0.0060 & 0.0038 & 80.6674 & 0.0268 & 0.4185 & 1 & Infinity \\
4 & 1.7472 & 0.3222 & 0.0060 & 0.0038 & 80.6674 & 0.0268 & 0.4185 & 1 & 0.0000 \\
5 & 1.7472 & 0.3222 & 0.0060 & 0.0038 & 80.6674 & 0.0268 & 0.4185 & 1 & 0.0000 \\
6 & 1.7472 & 0.3222 & 0.0057 & 0.0048 & 80.6674 & 0.0413 & 0.4173 & 1 & 0.2092 \\
7 & 1.7472 & 0.3222 & 0.0057 & 0.0048 & 80.6674 & 0.0413 & 0.4173 & 1 & 0.1195 \\
8 & 1.7472 & 0.3222 & 0.0061 & 0.0020 & 80.6674 & 0.0509 & 0.4083 & 1 & 0.1474 \\
9 & 1.7472 & 0.3222 & 0.0061 & 0.0020 & 80.6674 & 0.0509 & 0.4083 & 1 & 0.0000 \\
10 & 1.7472 & 0.3222 & 0.0061 & 0.0020 & 80.6674 & 0.0509 & 0.4083 & 1 & 0.5350 \\
11 & 1.7472 & 0.3222 & 0.0081 & 0.0045 & 80.0080 & 0.0928 & 0.4001 & 1 & 0.4996 \\
12 & 1.7472 & 0.3222 & 0.0081 & 0.0045 & 80.0080 & 0.0928 & 0.4001 & 1 & 0.0324 \\
13 & 1.7472 & 0.3222 & 0.0081 & 0.0045 & 80.0080 & 0.0928 & 0.4001 & 1 & 0.1473 \\
14 & 1.7472 & 0.3222 & 0.0081 & 0.0045 & 80.0080 & 0.0928 & 0.4001 & 1 & 0.0000 \\
15 & 1.7212 & 0.3222 & 0.0063 & 0.0037 & 79.6674 & 0.1039 & 0.3963 & 1 & 0.0794 \\
16 & 1.7212 & 0.3222 & 0.0063 & 0.0037 & 79.6674 & 0.1039 & 0.3963 & 1 & 0.0000 \\
17 & 1.7212 & 0.3222 & 0.0063 & 0.0037 & 79.6674 & 0.1039 & 0.3963 & 1 & 0.9126 \\
18 & 1.7212 & 0.3222 & 0.0063 & 0.0037 & 79.6674 & 0.1039 & 0.3963 & 1 & 0.1473 \\
\hline
\end{tabular}

Generally, there is a need for a simple and at the same time reliable criterion for assessing the level of trustworthiness in selecting the best solution among those located in the first Pareto front. The crowding distance criterion routinely being used in NSGA-II seems to be sufficient, but it is a pure mathematical measure. Hence, depending on the specific engineering problem to be addressed, one may need more practical criteria. For instance, in the current problem, it is apparent that one would need to identify solutions for which the summation of errors in both objectives took a minimum value. Thus, although 18 solutions obtained are all acceptable from a purely mathematical measure point of view, they are identified and sorted on the basis of least total error. Thus, the first solution is identified as the best with a minimum sum of errors in MWD and CCD profiles. The errors in MWD and CCD patterns are 0.110513 and $0.163938 \%$, with the total error of $0.274451 \%$. 
The copolymerization recipe corresponding to this solution (first row of Table 4) is suggesting $1.7212,0.3222,0.0081$, and $0.0020 \mathrm{~mol} \mathrm{~L}^{-1}$, respectively, as optimal concentrations for ethylene, 1-butene, hydrogen, and cocatalyst, to be used in the synthesis of the target copolymer defined as Case I, at the polymerization temperature of $80^{\circ} \mathrm{C}$. The final optimal copolymerization recipe is very close to the one identified initially and utilized to calculate/construct the target MWD and CCD patterns fed into the NSGAII code as inputs (Table 3). Obviously, the optimizer has been quite successful in reproducing satisfactorily (acceptably in practical terms) the same recipe in the reverse pathway.

In a similar manner, the NSGA-II code identified after 9,403 iterations the first Pareto front for the target copolymer defined as Case II in Table 3. In this regard, the maximum allowable error in the simultaneous optimization of MWD and CCD patterns was arbitrarily set to be $0.02 \%$. The Pareto front of this case together with variation of errors in the optimization of MWD and CCD profiles in terms of epoch, individually and cumulatively, are plotted in Figure 5. It was found that only one among 314 solutions located in the first Pareto front met the criterion of maximum error of $0.02 \%$, with 0.019901 and $0.002867 \%$ errors in the optimization of the MWD and CCD patterns, and total error of $0.022768 \%$. Similar to what happened in the case of target copolymer I, the copolymerization recipes obtained were close to each other. The optimum copolymerization recipe proposed by the NSGA-II code for the production of target copolymer II was ethylene concentration of $1.012782 \mathrm{~mol} \mathrm{~L}^{-1}$, 1-butene concentration of $0.030382 \mathrm{~mol} \mathrm{~L}^{-1}$, hydrogen concentration of $0.008090 \mathrm{~mol} \mathrm{~L}^{-1}$, cocatalyst concentration of $0.003001 \mathrm{~mol} \mathrm{~L}^{-1}$, and temperature of $89.99997^{\circ} \mathrm{C}$. These are again very close to the quantities manually set to construct target MWD and CCD patterns (as per Table 3). 
(A)

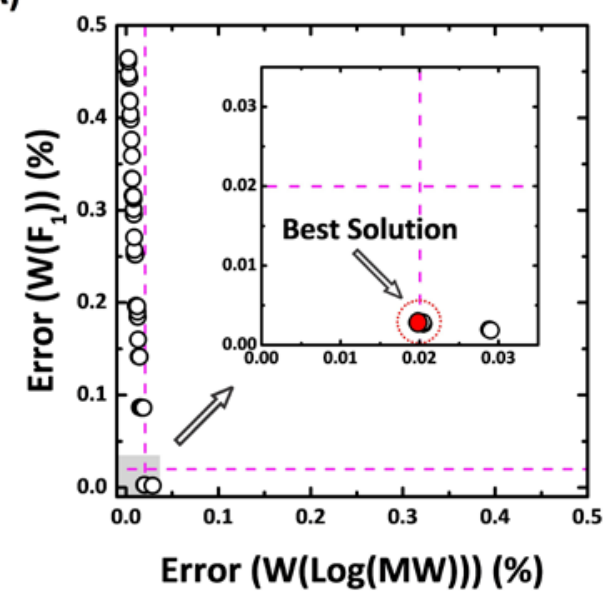

(B)

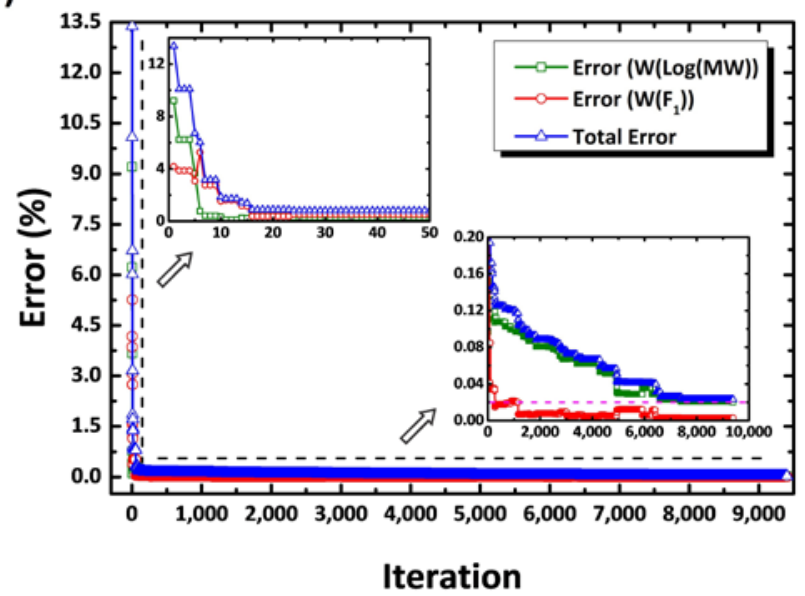

Figure 5. The Pareto optimal front (A) and errors in prediction of MWD and CCD patterns (B) proposed by the NSGA-II code for Case II target copolymer.

Figure 6 compares the microstructural patterns of the best solution proposed by the NSGA-II optimizer (green symbols) with the theoretical patterns yielded from summation equations 1 and 2 (blue curves) and those considered by the code for the target copolymer II (red lines). Again, the obtained results verify the reliability of the multiobjective optimization process using the developed NSGA-II code. Based upon the NSGAII code results with the microstructural patterns of two target copolymers arbitrarily selected with known copolymerization recipes, the simulator can be put to practice in order to identify/select the best copolymerization recipe needed for synthesizing/tailoring ethylene/ $\alpha$-olefin copolymers with predefined microstructural patterns. 

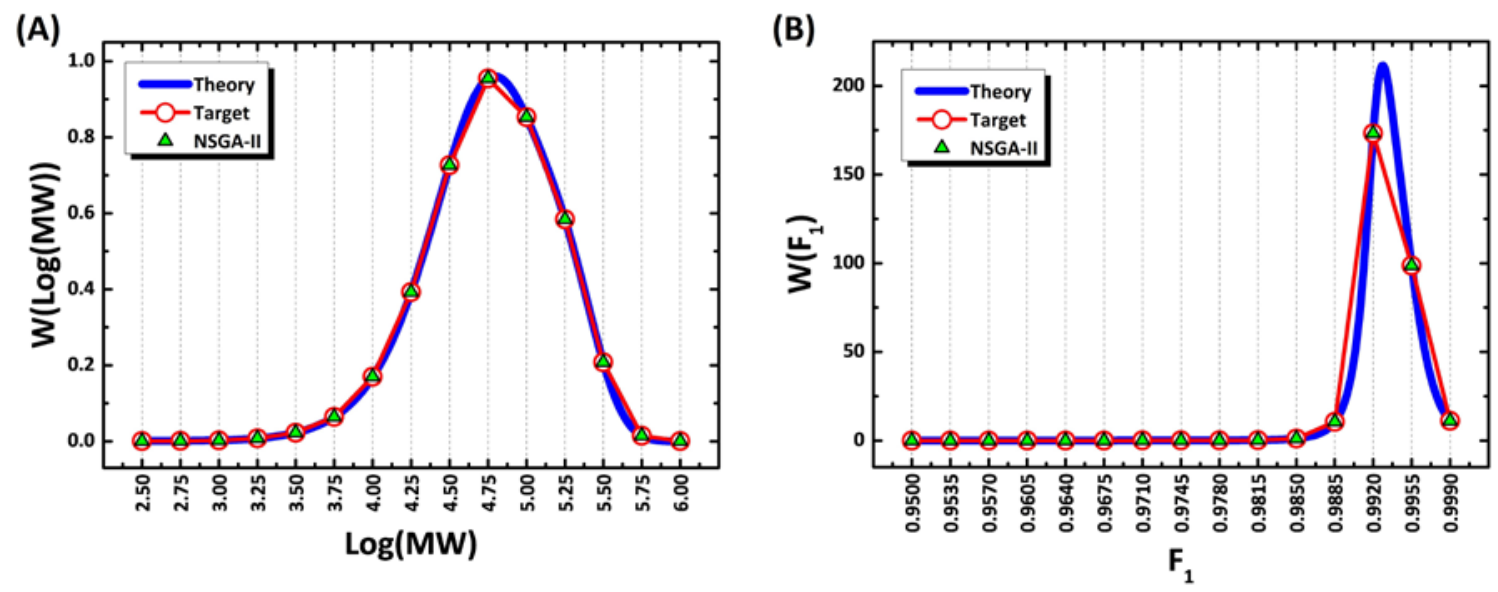

Figure 6. Comparison of the optimization results (NSGA-II) and predefined targets (theoretical plots obtained using summation equations 1 and 2) in case of copolymer II; MWD (A) and CCD (B) patterns.

\subsection{Implementation of NSGA-II optimizer in synthesis of desirable copolymers}

In the previous section it has been confirmed that the developed optimization tool is capable of receiving microstructural patterns (i.e., MWD and CCD profiles discretized over 30 points as 30 objectives) and finding the best copolymerization recipe, consisting of 5 optimal levels of operating variables to be set for the realization of the target copolymers. In this part, two desirable copolymers with arbitrary MWD and CCD profiles are defined to challenge further the performance of the developed NSGA-II optimization tool. Table 5 cites the distribution patterns of molecular weight and copolymer composition of the defined desirable copolymers, from which 30 equally-spaced points are specified as inputs for the NSGA-II optimizer. As visualized earlier in Scheme 1, the NSGA-II optimizer explores the preset search space and recalls summation equations 1 and 2 along with kinetic parameters in order to calculate and analyze the fitness of chromosomes (utilizing operators of the genetic algorithm) and eventually find the best solutions for each desirable case. In contrast to cases studied in the previous section, the copolymerization recipes are now unknown. For the desirable copolymer defined as Case III in Table 5, the NSGA-II optimizer searched over thousands of scenarios in order to locate the most similar MWD and CCD patterns among all possible scenarios through multi-objective 
optimization based on 30 target values or objectives. In this case, the optimization process is set to stop whenever the error values for the best member of the evolving population remain unchanged for at least 100 consecutive iterations. 17 solutions were separated as the optimum solutions located in the first Pareto front after 5,473 iterations, and the solution with minimum total error value was reported as the best solution. The errors for recognition of the target MWD and CCD patterns by the best solution were $4.933 \%$ and $7.857 \%$, respectively. The corresponding optimum copolymerization recipe proposed by the NSGA-II code for the production of target desirable copolymer III was ethylene concentration of $3.106811 \mathrm{~mol} \mathrm{~L}^{-1}$, 1-butene concentration of $0.132823 \mathrm{~mol} \mathrm{~L}^{-1}$, hydrogen concentration of $0.000860 \mathrm{~mol} \mathrm{~L}^{-1}$, cocatalyst concentration of $0.004096 \mathrm{~mol} \mathrm{~L}^{-1}$, and temperature of $86.47378{ }^{\circ} \mathrm{C}$. The microstructural profiles of the best solution along with the target copolymer microstructural profiles, are shown in Figure 7 for copolymer III. The green symbols represent the best values among all possibilities checked and proposed by the optimizer considering the preset search space for input variables. Also, the corresponding theoretical microstructural patterns (blue curves) are constructed by recalling summation equations 1 and 2 for the best recipe in view of the kinetics of ethylene/1-butene copolymerization. It is worth mentioning here that the errors calculated/reported in this work do not reflect the precision of the applied intelligent optimization method. In fact, the word 'error' shows/quantifies the amount of deviation for each phenotype (i.e., the microstructural patterns corresponding to each genotype or recipe) from the target input microstructural profiles. In fact, the optimizer not only meticulously explores and identifies all the best possible solution(s) for any given set of target microstructural patters, but also precisely computes and reports the exact amount of the deviation from the predefined target.

Table 5. MWD and CCD patterns of two desirable copolymers defined and utilized to challenge the performance of the multi-objective optimization tool. 


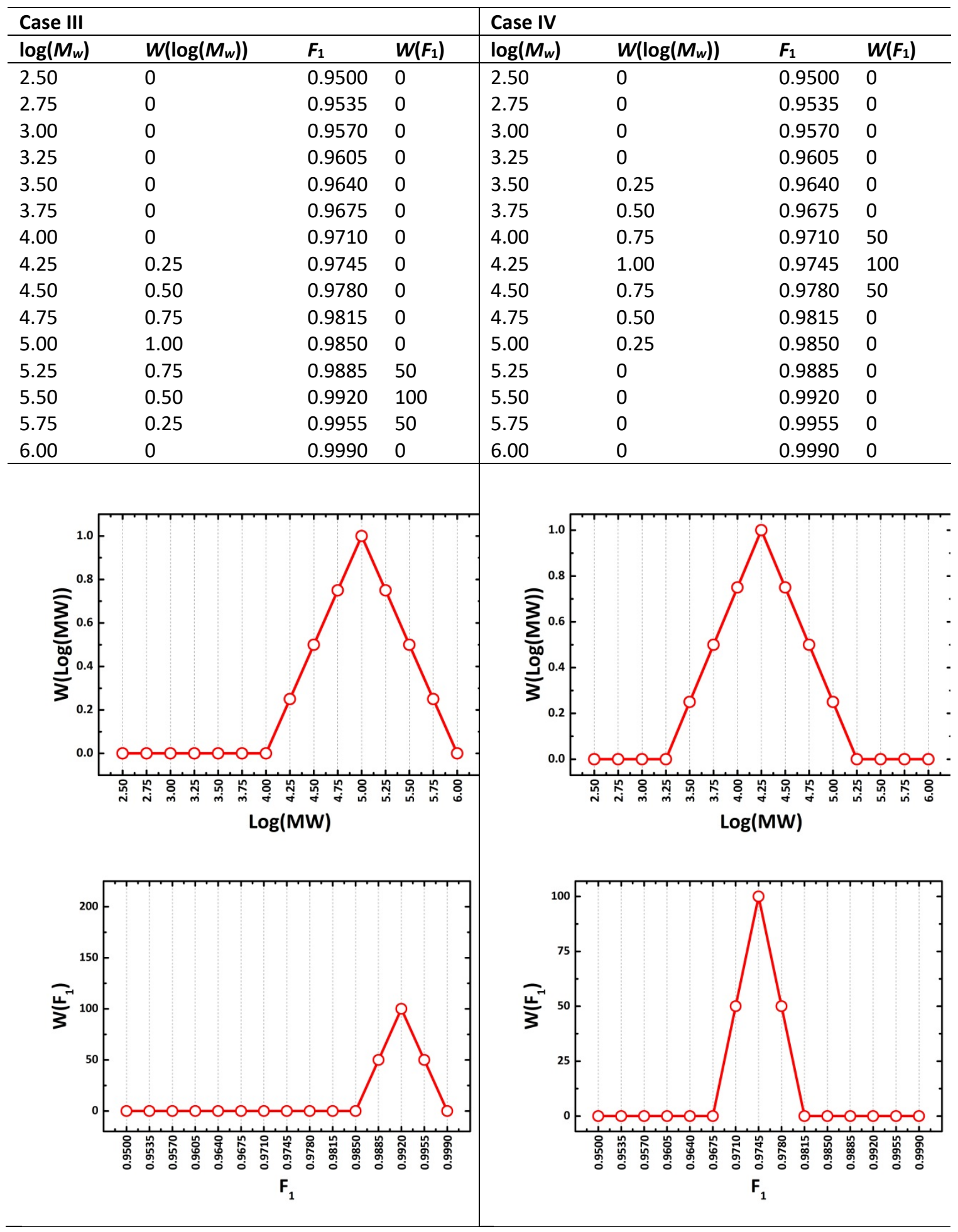

In the case of desirable copolymer IV, 249 solutions were obtained after 8,073 iterations. In this case, the errors reported for recognition of the target MWD and CCD patterns by the best solution were $16.317 \%$ and $16.782 \%$, respectively. Furthermore, the optimum 
copolymerization recipe was ethylene concentration of $0.318207 \mathrm{~mol} \mathrm{~L}^{-1}$, 1-butene concentration of $0.035784 \mathrm{~mol} \mathrm{~L}^{-1}$, hydrogen concentration of $0.009655 \mathrm{~mol} \mathrm{~L}^{-1}$, cocatalyst concentration of $0.013206 \mathrm{~mol} \mathrm{~L}^{-1}$, and temperature of $88.05094{ }^{\circ} \mathrm{C}$. The MWD and CCD patterns of the best solution (green symbols) along with the target copolymer microstructural profiles (red lines), are shown in Figure 8 for copolymer IV. Interestingly, although a unimodal MWD profile has been defined for desirable copolymer IV, the optimization results proposed a bimodal MWD pattern. Undoubtedly, this is dictated by the copolymerization kinetics and the nature of recipe-microstructure interrelationships (summation equations 1 and 2), along with the range of variations (potential search spaces) preset for compositional and operational conditions. Since the NSGA-II optimizer needs to satisfy 30 objectives at the same time, one cannot expect to obtain always a unimodal profile for MWD or CCD patterns by the optimizer. However, the unimodal profile suggested by the NSGA-II optimizer for MWD in the case of desirable copolymer IV is close to the bimodal profile proposed by Eq. (1). Thus, the developed NSGA-II optimizer can find the 'best' microstructural patterns for any desired pair of MWD and CCD.

\section{CONCLUSION}

Regulating the balance between the elements of the triplet polymer processingproperties-price is part of the Engineering art. In case of coordination copolymerization, difficulties are mainly due to the inability of exactly tuning the efficiency and characteristics of multi-site catalytic systems. Therefore, the resulting copolymers may exhibit a range of properties depending on feed composition, catalyst combination, and operating conditions, including recipe component concentrations and polymerization temperature. 
(A)

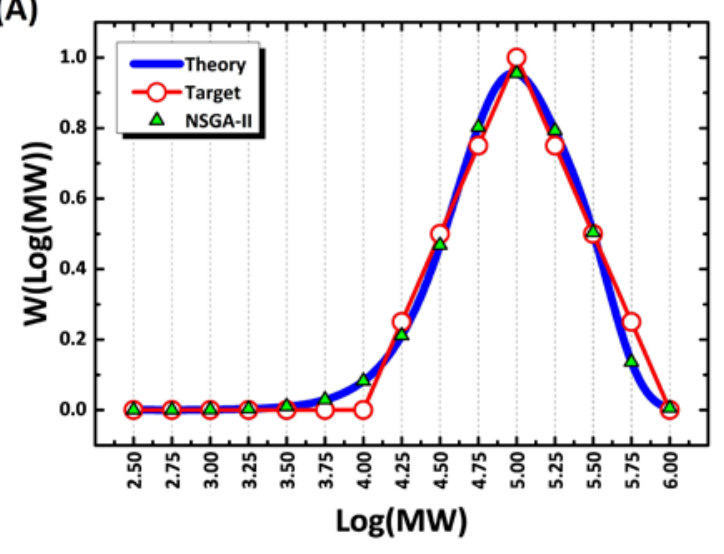

(B)

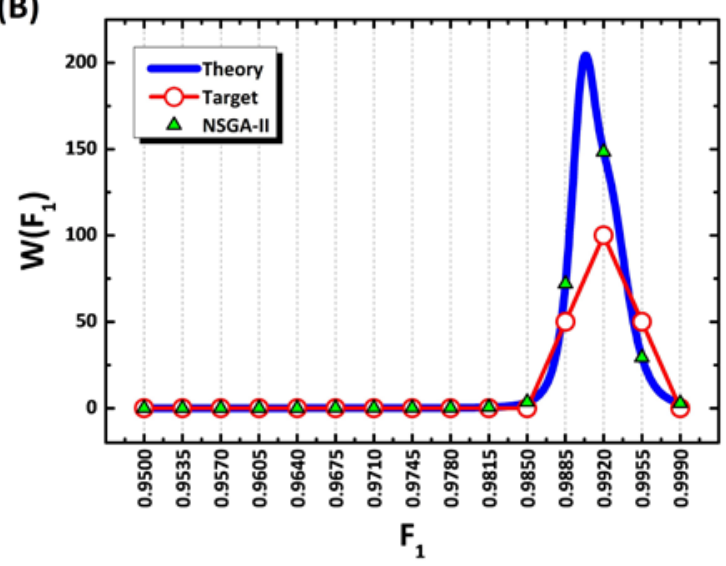

Figure 7. Comparison of the optimization results (NSGA-II) and predefined targets (theoretical plots obtained using summation equations 1 and 2 ) in case of desirable copolymer III; MWD (A) and CCD (B) patterns.

(A)

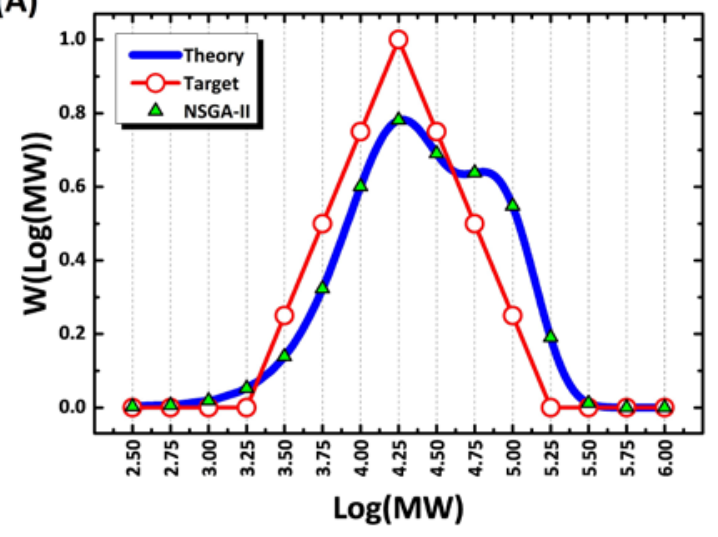

(B)

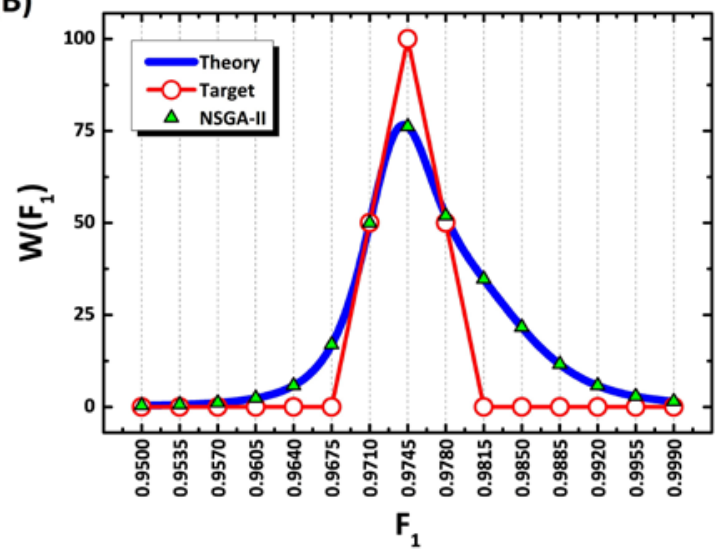

Figure 8. Comparison of the optimization results (NSGA-II) and predefined targets (theoretical plots obtained using summation equations 1 and 2 ) in case of desirable copolymer IV; MWD (A) and CCD (B) patterns.

There is need, however, for synthesizing copolymers with desired microstructures for target applications. In this sense, the three vertices of the polymer processing-propertiesprice triangle should be designed and delivered appropriately, for the length and angle between them can be determined by application considerations. 
Artificial Intelligence methodology was applied to recognize copolymer microstructural patterns and identify optimal copolymerization recipes for synthesizing predefined copolymers. A computer code was developed based on NSGA-II that receives desirable MWD and CCD profiles and suggests the best copolymerization recipe. Metallocenecatalyzed copolymerization of ethylene with 1-butene was chosen as a typical case study in view of complexities associated with multiple catalytic systems and availability of reliable experimental data and modeling equations for prediction of MWD and CCD patterns in terms of operating conditions, including polymerization temperature and concentrations of feed components. It was shown that the developed optimizer can explore and find the best copolymerization recipe needed for the production of target ethylene/1-butene copolymers, as signaled by negligible differences between the optimizer outputs and the preset targets, i.e., for case studies with known recipemicrostructure interrelationships. Even more interestingly, the optimizer proposed the best MWD and CCD profiles when it was fed by desirable copolymer microstructures, which is a very unique feature of this work.

\section{REFERENCES}

[1] J.-F. Lutz, J.-M. Lehn, E. W. Meijer, K. Matyjaszewski, Nature Reviews Materials, 2016, 1,1 .

[2] J. B. P. Soares, Chemical Engineering Science, 2001, 56(13), 4131.

[3] M. R. Saeb, Y. Mohammadi, A. S. Pakdel, A. Penlidis, Macromolecular Therory and Simulations, 2016, 25(4), 369.

[4] M. R. Saeb, Y. Mohammadi, H. Rastin, T. S. Kermaniyan, A. Penlidis, Macromolecular Therory and Simulations, 2017, 26(5), DOI: 10.1002/mats.201700041.

[5] Y. Mohammadi, M. Najafi, V. Haddadi-AsI, Macromolecular Therory and Simulations, 2005, 14(5), 325.

[6] W. Stockmayer, The Journal of Chemical Physics, 1945, 13, 199.

[7] B. M. Shaw, K. B. McAuley, D. W. Bacon, Polymer Reaction Engineering, 1998, 6(2), 113. 
[8] N. R. Sturtevant, V. Bulitko, Journal of Artificial Intelligence Research, 2016, 57, 307.

[9] K. Muthukumar, S. Jayalalitha, International Journal of Electrical Power \& Energy Systems, 2016, 78, 299.

[10] J. W. Simpson-Porco, F. Bullo, IEEE Transactions on Smart Grid, 2016, 7(4), 1979.

[11] D. M. D'Addona, R. Teti, Procedia CIRP, 2013, 7, 323.

[12] J. Sjöberg, Q. Zhang, L. Ljung, A. Benveniste, B. Delyon, P.-Y. Glorennec, H. Hjalmarsson, A. Juditsky, Automatica, 1995, 31(12), 1691.

[13] F. A. N. Fernandes, L. M.F. Lona, Brazilian Journal of Chemical Engineering, 2005, 22(3), 401.

[14] J. Zhang, Chemical Engineering Science, 2008, 63(5), 1273.

[15] J. Zhang, A.j. Morris, E.B. Martin, C. Kiparissides, Chemical Engineering Journal, 1998, 69(2), 135.

[16] A. d'Anjou, F. J. Torrealdea, J. R. Leiza, J. M. Asua, G. Arzamendi, Macromolecular Theory and Simulations, 2003, 12(1), 42.

[17] C. W. Ng, M. A. Hussain, Chemical Engineering and Processing: Process Intensification, 2004, 43(4), 559.

[18] J. C. B. Gonzaga, L. A. C. Meleiro, C. Kiang, R. Maciel Filho, Computers \& Chemical Engineering, 2009, 33(1), 43.

[19] R. J. Minari, G. S. Stegmayer, L. M. Gugliotta, O. A. Chiotti, J. R. Vega, Macromolecular Reaction Engineering, 2007, 1(3), 405.

[20] T. Charoenpanich, S. Anantawaraskul, J. B. P. Soares, Macromolecular Reaction Engineering, 2016, 10(3), 215.

[21] J. D. Kim, J. B.P. Soares, G. L. Rempel, Journal of Polymer Science Part A: Polymer Chemistry, 1999, 37(3), 331.

[22] S. Chakravarti, W. H. Ray, Journal of Applied Polymer Science, 2001, 80(8), 1096.

[23] P. K. Shukla, K. Deb, S. Tiwari, International Conference on Evolutionary MultiCriterion Optimization, 2005, 311. 
[24] D. Greiner, B. Galván, J. Periaux, N. Gauger, K. C. Giannakoglou, G. Winter, Advances in Evolutionary and Deterministic Methods for Design, Optimization and Control in Engineering and Sciences, Springer International Publishing, Switzerland, 2015.

[25] S. Garshasbi, J. Kurnitski, Y. Mohammadi, Applied Energy, 2016, 179, 626.

[26] B. Baghaei, M. R. Saeb, S. H. Jafari, H. A. Khonakdar, B. Rezaee, V. Goodarzi, Y. Mohammad, Journal of Applied Polymer Science, 2017, 134(33), 45145.

[27] R. Azari, S. Garshasbi, P. Amini, H. Rashed-Ali, Y. Mohammadi, Energy and Buildings, 2016, 126, 524.

[28] S. Kannan, S. Baskar, J. D. McCalley, P. Murugan, IEEE Transactions on Power systems, 2009, 24(1), 454.

[29] M. Hosseinnezhad, M. R. Saeb, S. Garshasbi, Y. Mohammadi, Solar Energy, 2017, 314. 